\title{
Alexandra David-Néel ou l'art de la fugue et du déguisement
}

Manuela Ledesma Pedraz

\section{(2) OpenEdition \\ 1 Journals}

Édition électronique

URL : https://journals.openedition.org/clio/8712

DOI : $10.4000 /$ clio.8712

ISSN : 1777-5299

Éditeur

Belin

Édition imprimée

Date de publication : 15 décembre 2008

Pagination : 213-22

ISSN : 1252-7017

Référence électronique

Manuela Ledesma Pedraz, « Alexandra David-Néel ou l'art de la fugue et du déguisement », Clio.

Histoire, femmes et sociétés [En ligne], 28 | 2008, mis en ligne le 15 décembre 2011, consulté le 22 avril 2022. URL : http://journals.openedition.org/clio/8712 ; DOI : https://doi.org/10.4000/clio.8712 


\title{
Alexandra David-Néel ou l'art de la fugue et du déguisement
}

\author{
Manuela LEDESMA PEDRAZ
}

Marine Lorrain : Dans votre travesti, est-ce que vous pouviez compter sur une aide quelconque des Tibétains tout au long de votre pèlerinage? Comment faisiez-vous pour subsister?

Alexandra David-Néel : Ah, il y avait des villages. Devant les villages, on s'en allait mendier, puisqu'on était des mendiants. On s'en allait mendier et, puis, on chantait des choses religieuses aux portes des villages. Je fais ça très bien, du reste; mon fils aussi, lui, il est tibétain, naturellement. Et alors, on récoltait des aumônes. Oh, quelquefois c'était pas très bon, d'autres fois c'était à peu près mangeable. Et puis, quand on n'en avait pas, et bien, ma foi, on jeûnait. Ça arrive, ces choses-là. Il nous est arrivé de rester six jours sans manger. Il n'y avait plus rien, nous étions dans les neiges, absolument perdus. On a traversé des montagnes alors enfouis dans la neige. Et il neigeait, il neigeait, il neigeait, il a neigé comme ça pendant ces six jours. Enfin, nous avons trouvé une espèce de hutte qui était habitée pendant l'été par des pasteurs qui montaient jusque-là avec leurs troupeaux. À ce moment-là, il y avait encore une provision de bouses de vaches séchées dans un coin de cette hutte. Ça nous permettait de faire du feu et de faire fondre de la neige pour avoir de l'eau, mais il n'y avait rien à manger. Et alors, vous savez ce que nous avons fait? Il nous restait dans notre sac un morceau de peau qui nous servait à ressemeler nos bottes parce que c'est des chaussures tibétaines avec des semelles en peau non tannée et tout à fait légère, alors on doit souvent les 
ressemeler. Il en restait un morceau. Alors, ce morceau on l'a fait bouillir. Ça fait du bouillon, n’est-ce pas ? Ça se mange ! Enfin, les jours suivants, tout de même, nous avons rencontré des gens qui nous ont donné du thé. Il était temps, tout de même !

Marine Lorrain: Et ces gens que vous rencontriez, par exemple, vous savaient blancs et mendiants?

Alexandra David-Néel : Ah! Pas du tout qu'ils nous savaient pas blancs. Ah si..., nous aurions été bienvenus! [sic]. On ne nous aurait pas tués, non. On nous aurait ramenés au premier magistrat venu pour qu'on nous remette à la frontière. D’abord, mon fils n'était pas blanc, il était tibétain, lui. Il n'y avait que moi. Moi, j'étais la vieille mère. Naturellement, j'étais beaucoup plus jeune que maintenant, mais enfin je n'étais pas très jeune. Mais alors j'imitais tout à fait la vieille caduque et tout ce qu'il y a de plus misérable. Alors, qui aurait pensé que c'était une femme blanche ? Je parle très bien tibétain et puis je sais très bien me conduire en tibétain. Et puis, alors, n'est-ce pas, on ne se lavait pas, alors on était un peu bruns, tout de même. Alors, on était très respectables. Nous faisions un pèlerinage, c'était très bien. Nous étions des gens très pieux, très respectés, et puis le lama [son fils] est un lama somme toute, et un lama instruit. Alors, il lisait les livres religieux. Il prêchait à ces gens-là des sermons très édifiants. Ça allait très bien.

Voyez-vous, au Tibet, c'est comme partout : il y a des riches et des pauvres. Et même chez les paysans : il y a des paysans riches qui ont beaucoup de bétail, qui ont des terres assez considérables, et puis il y a les paysans minables. Alors, chez les paysans minables, eh bien, la vie est assez restreinte. On mange de la tsampa, c'est-à-dire de la farine d'orge grillée ou du thé, et puis très rarement de la viande. Chez les paysans riches, au contraire, la maison est confortable, suivant leur idée du confort, et alors là, ils ont de la viande séchée, du fromage, du beurre, du thé en quantité et, ma foi, la vie est très agréable.

Marine Lorrain : D'où, au fond, le Tibétain est accueillant pour l'étranger qui frappe à sa porte? 
Alexandra David-Néel : Eh, eh... Ici, il faut ouvrir une parenthèse. Ils sont accueillants... Ah, mais pas toujours! Non, non. Voyez-vous, il y a, à propos des voyageurs, une superstition au Tibet. On dit que les voyageurs sont souvent suivis pas des démons. Ces démons pénètrent dans les maisons où les voyageurs sont accueillis. Et alors, s'il y a des gens faibles, des gens malades, alors ces démons, dont la spécialité est de se nourrir des souffles - vous diriez peut-être l'âme, vous -, enfin du souffle vital des gens, ces démons font leur proie de ces être faibles ou de ces êtres malades. Alors, comme les Tibétains croient à cela, ils se méfient très fort des voyageurs parce qu'ils craignent les démons qu'ils traînent à leur poursuite. Si bien que, souvent, s'il y a un malade dans une maison, on vous ferme la porte au nez et on vous dit: " on n'entre pas, on n'entre pas ». Et on vous laissera là, dehors, à la proie des chiens quand il y en a. Maintenant... Oh, ce n'est pas toujours comme ça, moi j’ai souvent été très bien accueillie dans les maisons et j’y ai demeuré avec les gens, j’ai pu les voir vivre de près. Mais j'avais déjà vu tout cela avant ce voyage-là. J'étais parfaitement au courant de la vie des Tibétains.

Archives sonores de la Radio Suisse Romande, Radio-Genève, MP 56.101, 4 août 1956 (enregistrement), 21 janvier 1957 (diffusion): Entretien avec Alexandra David-Néel, la plus grande exploratrice de l'Orient (1/6) : Voyage à Lhassa, la ville interdite $d u$ Tibet. Interviewée par Marine Lorrain, collaboratrice Radio-Genève. Durée du document: 22'24". Durée de l'extrait : 4'46". Cet extrait est publié dans : Entretiens avec Alexandra DavidNeel, Tibet, Inde, Chine, RSR et Éditions Zoé, 2006 (2 CD, 1 livret).

En août 1956, âgée de 88 ans, Alexandra David-Néel, première femme occidentale à être entrée à Lhassa en 1924, accorde une série d'entretiens à Radio-Genève sur le Tibet, l'Inde et la Chine. D'une voix toujours enthousiaste malgré son âge, elle y raconte ses faits et prouesses pendant ses années de voyage en Asie, signe que la mémoire de l'orientaliste en a déjà fait le tri et n'a gardé que les aspects les plus exaltants de ses aventures. Si nous avons décidé de commenter cet extrait où elle parle de l'accueil qui lui avait été fait par les Tibétains pendant son voyage au Pays des Neiges après trois tentatives infructueuses, c'est pour deux raisons. Premièrement, à cause de l'attraction mystérieuse que ce pays exerçait sur elle, ce qui nous 
permettra d'aborder la question du désir de rupture et de perdition ${ }^{1}$ qui détermine son projet ; deuxièmement, parce que ce voyage n'a été possible que par l'entremise du déguisement, condition sine qua non de son succès. Ce fait nous renvoie à la question, essentielle ici, de la confrontation du sujet qui voyage à l'expérience de l'autre sous toutes ses formes, c'est-à-dire à la problématique de l'altérité. Étant donné que cette expérience est à l'origine d'un récit intitulé Voyage d'une Parisienne à Lhassa (1927), ce sont ces deux composantes du récit de voyage au $\mathrm{XX}^{\mathrm{e}}$ siècle que nous allons commenter, tout en nous appuyant sur les lettres écrites à son mari pendant ce temps-là. Il faut également signaler que cet ouvrage a été le premier écrit par Alexandra David-Néel avec la volonté explicite et avouée d'atteindre par là à l'indépendance économique, car cette voyageuse qui voulait devenir une orientaliste reconnue s'attelle, une fois de retour en France, à la tâche d'écrire, convaincue que seul l'affranchissement économique de la femme peut la rendre autonome ${ }^{2}$, donc libre.

Stimulée dès sa petite enfance, ainsi qu'il est d'usage parmi les écrivains voyageurs, par la contemplation d'un atlas offert par son père à l'âge de six ans et par la lecture des livres de Jules Verne depuis sa septième année, Alexandra David-Néel, qui avait toujours fait preuve d'une grande force de caractère et s'était initiée très jeune aux philosophies orientales, réussit à entreprendre le grand voyage dont elle rêve depuis longtemps en 1911, à quarante-trois ans, transgressant ainsi les normes établies par une société qui vise à maintenir les femmes au foyer ${ }^{3}$. Avec le consentement plus ou moins acquis de son mari, qui la finance, elle précise pourtant le sens de ses voyages au fur et à mesure qu'ils se déroulent aussi bien dans le temps que dans l'espace. C'est

Voir à ce sujet Cogez 2004 : 11-33.

2 Au début du XXe siècle, Alexandra David-Néel avait déjà écrit, sous le nom d'Alexandra Myrial, dans différents journaux et revues, dont La Fronde, des articles sur la condition des femmes. Elle reprend et amplifie ces idées dans une brochure intitulée Féminisme rationnel, parue en 1909. Texte repris dans David-Néel 2003.

3 Sans oublier que, en 1891, à vingt-trois ans, elle avait déjà fait son premier voyage en Inde, faisant alors preuve d'un esprit d'aventure et d'une audace étonnants à l'époque chez une jeune femme, car non seulement elle fait le voyage toute seule, mais elle se débrouille aussi parfaitement dans ce pays inconnu pendant quelque dix-huit mois. Voir David-Néel 1951. 
ainsi que son rêve initial et assez flou de l'Asie devient, au cours du long voyage entrepris en 1911 et terminé en 1925, le rêve du Tibet. Autrement dit, puisque le voyage « se passe de motifs » et «se suffit à lui-même $»^{4}$, sa hantise de pénétrer coûte que coûte dans ce pays alors interdit se fait jour petit à petit.

Ayant toujours été, d'après elle-même, une sauvage à tendances mystiques, ce grand voyage est déterminé chez Alexandra David-Néel par un profond désir de rupture par rapport au monde occidental, qu'elle considère superficiel et vide de sens, dont elle avoue abhorrer aussi bien les conventions que le grégarisme. Il s'agit de s'éloigner d'un monde dit civilisé qui l'asphyxie, au profit, bien entendu, de la réalisation de ses « rêves de petite fille sauvage ${ }^{5}$. Car Alexandra avoue ouvertement dans ses lettres préférer, à la vie dans les grandes villes de l'Occident, la solitude et le silence au milieu des grands espaces, voire « la rude vie, sous la tente, des nomades de l'Asie centrale $»^{6}$. Or, c'est précisément au Tibet, aussi bien par l'éblouissement que lui procurent les paysages grandioses qu'elle parcourt que par la découverte de la philosophie et des pratiques lamaïstes apprises en cours de route, qu'elle croit avoir trouvé la place qui lui correspond en ce monde. Rien n'y fait alors pour la détourner de son but, ni la maigre nourriture ni le jeûne forcé, ni le froid extrême ni la neige effaçant des jours durant les traces du chemin. L'appel de cet ailleurs presque féerique est si fort et si intime qu'il finit par se doubler ici d'un voyage au fond de soi-même, devenant par là un véritable voyage initiatique qui conduit notre voyageuse à la transformation personnelle, ce qui peut très bien se traduire par un désir de perdition au sein de la terre élue :

À l'heure actuelle, quelques raisonnements que je puisse me faire et, ce qui est plus fort que tous raisonnements, quel que soit mon désir de te revoir [...], je ne puis pas m'arracher au Tibet. Il y a là un phénomène étrange, que je ne m'explique pas. Je suis liée, clouée, il n'y a pas d'effort de volonté qui tienne; si je m'arrache, c'est ma vie que j'arracherais. [...] Atavisme

\footnotetext{
Bouvier $2001: 12$.

David-Néel 1975 : 232. Lettre du 12 décembre 1912.

6 David-Néel 1976 : 52. Lettre du 5 octobre 1918.
} 
lointain ou souvenir de vies antérieures, comme dirait un hindou. Mystère ! ${ }^{7}$

Ce mystère, elle l'attribue à ses ancêtres maternels et, plus précisément, au père de sa mère, dont la mère était asiatique. Non sans une certaine coquetterie, elle parle de «la goutte de sang mongol qui a soudain repris vie après plusieurs générations », avouant qu'elle a «cela dans le sang» et qu'elle rêvait «les mêmes rêves » quand elle avait six $a^{8}{ }^{8}$. Quoi qu'il en soit, c'est l'irrésistible attraction que le Tibet exerce sur Alexandra, ainsi que sa force de volonté et son esprit d'aventure, qui rendent possible la réalisation de son rêve de l'ailleurs asiatique et, particulièrement, son séjour de quatre mois au Tibet.

En ce qui concerne le déguisement comme stratégie pour y parvenir, nous voudrions signaler que, à la différence du travestissement, par lequel les femmes ont pu exprimer leur désir d'émancipation', le déguisement ne prétend qu'à l'identification avec l'autre. Dans le cas d'Alexandra, cela semble évident, mais ce procédé de mimétisation s'est fait par degrés. En effet, c'est en 1917, pendant son voyage au Japon, qu'Alexandra ressent pour la première fois la nostalgie d'un Tibet qu'elle n'a fait d'ailleurs qu'apercevoir en juillet 1916, une fois terminée sa retraite de vingt mois dans une caverne himalayenne auprès du Gomchen de Lachen, dont la philosophie et les pratiques lamaïques l'ont séduite. C'est pendant ce séjour au Japon qu'elle communique à son mari dans une lettre son "Grand Projet », lui parlant pour la première fois aussi de cette stratégie du déguisement. Du retour sur le continent asiatique, elle commence son périple à Pékin le 24 janvier 1918, arrive dans la ville-monastère de Koum-Boum, dans la province tibétaine de l'Amdo, fin juillet 1918, et y reste deux ans et demi entourée de 3800 moines, consacrés aussi bien à la méditation qu'à l'étude de textes bouddhiques de sources tibétaines. Elle quitte Koum-Boum le 5 février 1921 accompagnée de son fidèle Yongden (son fils adoptif), d'un domestique et de deux

David-Néel 1976 : 96. Lettre du 8 janvier 1920.

David-Néel 1976 : 116-117. Lettre du 26 juillet 1920.

9 Pensons, par exemple, à George Sand, ou, pour parler d'une écrivaine et voyageuse, à Isabelle Eberhardt, qui adopta le vêtement arabe masculin pour vivre au Sahara parmi les nomades. Voir à ce propos Pellegrin et Bard 1999. 
novices qui veulent voir du pays. L'orientaliste devient ainsi l'exploratrice du pays d'Amdo et du pays de Kham, dans le « Grand Tibet». Au départ, elle est revêtue de son bien mérité uniforme de dame-lama et passe pour une khandoma, «une promeneuse à travers l'espace $»^{10}$, mais, trop voyants, ces vêtements d'apparat seront bientôt remplacés par des vêtements chinois. Après trois échecs successifs pour se rendre à Lhassa11, la "Ville Soleil », elle se rend compte que c'est seule avec Yongden, sans bêtes ni bagages, qu'elle pourra pénétrer au pays interdit. Il lui faut donc se délester de tout ce qu'elle charrie encore du mode de vie occidental.

Fin septembre 1923, Alexandra et Yongden sont à Li-Kiang, la dernière ville chinoise du nord-est du Yunnan et, fin octobre, ils quittent le village de Tsédjrong sous prétexte de s'en aller herboriser dans la montagne, sur les premiers versants du Kha Karpo. Alexandra commence alors à mettre en place sa stratégie: elle laisse ostensiblement ses bagages au village, mais, étant arrivée en étrangère, elle est obligée, pour ne pas éveiller la méfiance des villageois, d'engager deux porteurs dont elle se débarrasse par la suite. Leurs bagages réduits au strict minimum, Alexandra et Yongden, enfin seuls, s'apprêtent à franchir le col de Dokar, qui marquait à l'époque la frontière du Tibet interdit, revêtant les habits des pèlerins tibétains, les ardjopas, «des moines pour la plupart - voyageant à pied, chargés de leurs bagages, qui, par milliers, errent à travers le Thibet [sic $»^{12}$. C'est donc ainsi que Yongden reprend le costume de lama et Alexandra, «dans le rôle de vieille mère ${ }^{13}$, se déguise en mendiante tibétaine. Il ne lui reste alors qu'à noircir ses cheveux et son visage pour parfaire son déguisement, ce qui est fait sur le champ au moyen de l'encre de chine pour les cheveux et, pour le visage, d'un «mélange de braise pulvérisée et de cacao ${ }^{14}$.

10 Expression utilisée par Jean Chalon 1985 : 380.

11 Brièvement, le premier échec a lieu à Foupien, où elle est reconnue et arrêtée le 21 juin 1921 ; le deuxième se produit sur la route de Lhassa, au mois de septembre de la même année ; le troisième, près des gorges de la Salouen, au printemps 1922.

12 David-Néel $1927: 38$.

13 David-Néel $1927: 39$.

14 David-Néel $1927: 40-41$. 
Or, le déguisement extérieur doit s'accompagner, pour réussir complètement, d'un processus nécessaire de mimétisation, ce qui dans le cas d'Alexandra n'est rendu possible que grâce à sa connaissance de la langue parlée par le peuple tibétain et de ses modes de comportement. Savoir qui a été accumulé par notre écrivaine au cours des trois dernières années de vie nomade, pendant lesquelles l'orientaliste est devenue non seulement l'exploratrice de « la dernière terre encore presque vierge $»^{15}$, mais aussi l'ethnographe qui pratique l'exercice de la curiosité des autres, consciente d'être, "parmi tous les voyageurs blancs, celui qui connaît le mieux le Tibet ${ }^{16}$. Le travail de mimétisation réussit à tel point qu'Alexandra et Yongden purent se promener partout au Tibet interdit pendant quatre mois en récoltant des aumônes avec le respect des gens, accueillis çà et là chez des paysans qui, riches ou pauvres, partagent avec eux leur nourriture, ne se doutant guère qu'ils ont affaire à une étrangère. Ainsi donc, ce voyage s'est révélé comme une véritable aventure de l'altérité. S'inspirant des données de l'ethnographie par son souci d'observation de la vie quotidienne, Alexandra David-Néel s'est confrontée à l'expérience de l'autre tibétain sous toutes ses formes, réussissant par là à s'assimiler complètement à cet autre que, par l'entremise des gènes transmis par sa mère, elle sent ressusciter en elle.

Arrivés à Lhassa le 28 janvier 1924, après avoir enduré des calamités et des fatigues sans nombre pendant ces quatre mois ${ }^{17}$, ils sont reçus par une tempête de sable, ce qui leur permet de ne pas être remarqués, mais, le premier éblouissement passé, c'est la déception qui s'installe dans l'esprit d'Alexandra. Voici, pour preuve, ce qu'elle raconte à ce propos dans une lettre écrite à son mari le 12 mars :

Je compte quitter Lhassa à bref délai. La ville est sans grand intérêt. Je suis rassasiée des visites aux lamaseries [...] En ville, les boutiquiers, en fait d'objets exotiques, étalent des piles de casseroles en aluminium... c'est

15 David-Néel 1976 : 78. Lettre du 4 mai 1919.

16 David-Néel 1976 : 238. Lettre du 29 février 1924 (date approximative).

17 Fatigues et calamités qui semblent être restées tout au fond de sa mémoire lors de l'entretien d'août 1956, mais qui avaient été minutieusement racontées dans ses écrits autobiographiques, c'est-à-dire dans les lettres à son mari. Voir, à ce propos, David-Néel 1975 et 1976. 
plutôt déconcertant. Mais je n'avais aucune curiosité au sujet de Lhassa. J'y suis allée parce que la ville se trouvait sur ma route et aussi parce que c'était une plaisanterie très parisienne à faire à ceux qui en interdissent l'accès. Ce qui m'a enchantée, c'est ma visite à ce qu'on peut appeler les vallées chaudes d'un pays froid. J'ai vu un Tibet inconnu des explorateurs, contemplé des paysages extraordinaires qui surpassent en splendeur tout ce que j'ai vu dans l'Himalaya et ailleurs... ${ }^{18}$

Au premier abord, il pourrait nous sembler choquant de constater le manque d'intérêt d'Alexandra pour la «Ville Soleil », ainsi que le fait qu'elle ne considère son exploit que comme une réponse au défi porté aux voyageurs par les autorités anglaises et chinoises, mais cela ne fait que mettre à nu que le véritable sens des voyages est dans le parcours et non pas dans le but atteint. Il est par ailleurs compréhensible que notre orientaliste se sente aussi déconcertée que déçue devant ces piles de casseroles en aluminium, qui ont dû lui faire l'effet d'une intrusion presque obscène dans ce monde qu'elle rêvait vierge. Heureusement pour elle, il y a eu ces vallées inconnues et son expérience de l'altérité absolue.

Alexandra David-Néel est donc bien ce «mythe intemporel » dont parle Nicolas Bouvier ${ }^{19}$, pour ses exploits et pour son courage, mais également pour son rôle de médiatrice entre le bouddhisme et le monde occidental, ce dont allaient se souvenir aussi bien des écrivains comme Allen Ginsberg et Jacques Kerouac que le philosophe et orientaliste Alan Watts. Quant aux voyages, son exemple fut brillamment suivi par Ella Maillart, exploratrice, dans la première moitié du XXe siècle, du Turkestan soviétique, de l'Afghanistan et du Népal.

\section{Bibliographie}

BOUvier Nicolas, 2001, L'usage du monde, Paris, Payot. Chalon Jean, 1985, Le lumineux destin d'Alexandra David-Néel, Paris, Perrin. Cogez Gérard, 2004, Les écrivains voyageurs au XXe siècle, Paris, Seuil. DAVID-NÉEL Alexandra, 1927, Voyage d'une Parisienne à Lhassa, Paris, Plon.

18 David-Néel 1976 : 237-238. Lettre du 28 février 1924 (date approximative).

19 Dans Maillart et Bouvier 2002. 
—, 1951, L'Inde où j'ai vécu, Paris, Plon.

—, 1975, Journal de voyage / 1. Lettres à son mari (11 août 1904-26 décembre 1917), Paris, Plon.

—, 1976, Journal de voyage/ 2. Lettres à son mari (14 janvier 1918-31 décembre 1940), Paris, Plon.

—, 1994, Voyages et aventures de l'esprit, Paris, Albin Michel

—, 2003, Féministe et libertaire. Écrits de jeunesse, Sens, Éditions Les nuits rouges.

Maillart Ella et Bouvier Nicolas, 2002, Témoins d'un monde disparu, Paris, Zoé.

Pellegrin Nicole et BARD Christine, 1999, "Introduction », Clio, Histoire, femmes et sociétés, 10, 1999. Femmes travesties : un « manvais » genre. 